\title{
Comparison of High-resolution Computerized Tomography with Film-screen Radiography for the Evaluation of Opacity and the Recognition of Coal Workers' Pneumoconiosis
}

\author{
Jingcai XING ${ }^{1,2, \dagger}$ Xiji HuAng ${ }^{1, \dagger}$, Lijuan YANG ${ }^{3}$, Yuewei $\mathrm{LiU}^{1}$, Hai ZHANG ${ }^{1}$ and \\ Weihong CHEN $^{1}$
}

${ }^{1}$ Key Laboratory of Environment and Health, Department of Occupational and Environmental Health, School of Public Health, Tongji Medical College, Huazhong University of Science and Technology, China, ${ }^{2}$ Department of Respiratory of the General Hospital of Xishan Coal \& Power Group, Co. Ltd., China and ${ }^{3}$ Department of Radiology of the General Hospital of Xishan Coal \& Power Group, Co. Ltd., China

\begin{abstract}
Comparison of High-resolution Computerized Tomography with Film-screen Radiography for the Evaluation of Opacity and the Recognition of Coal Workers' Pneumoconiosis: Jingcai XING, et al. Key Laboratory of Environment and Health, Department of Occupational and Environmental Health, School of Public Health, Tongji Medical College, Huazhong University of Science and Technology, China-Objectives: Current diagnostic standards of pneumoconiosis, which is the most serious occupational disease in China, are based on film-screen radiography (FSR). However, parenchymal structure overlap limits the sensitivity of FSR in the clinic. Highresolution computerized tomography (HRCT) has the advantage of identifying nodular pulmonary parenchymal changes. In this study, we aimed to compare HRCT and FSR for recognition of the profusion of small opacities, opacity shape and opacity coalescence in coal miners with or without radiographic evidence of coal workers' pneumoconiosis (CWP) and evaluate the possible role of HRCT in CWP diagnosis. Methods: There were 96 coal miners with radiographic evidence of CWP, 67 coal miners without CWP and 37 healthy controls in this study. FSR and HRCT images for all subjects were interpreted separately by three readers. Crude agreement and Kappa values were calculated to evaluate the agreement between the two methods. Results: The overall agreement for CWP evaluation was good (crude agreement rate $=87.1 \%, \kappa=0.72,95 \%$
\end{abstract}

Received Jul 3, 2013; Accepted May 7, 2014

Published online in J-STAGE Jun 21, 2014

Correspondence to: W. Chen, Key Laboratory of Environment and

Health, Department of Occupational and Environmental Health, School of Public Health, Tongji Medical College, Huazhong University of Science and Technology, Wuhan 430030, China (e-mail: wchen@mails.tjmu.edu.cn)

${ }^{\dagger}$ These authors contributed equally to this work.
Cl: 0.62-0.83) between FSR and HRCT in all coal miners. The sensitivity of HRCT for CWP diagnosis was $96.9 \%(93 / 96)$. We observed that 18 of the $67(26.9 \%)$ miners negative for CWP by FSR were classified as category 1 by HRCT according to the lung parenchyma profusion category system reported by Bérgin et al. The difference in the profusion scores between CWP subjects and healthy controls for HRCT scans were statistically significantly higher than those for FSR. Conclusions: HRCT was more sensitive than FSR in recognition of the profusion of small opacities among coal miners. More research is needed to evaluate the role of HRCT in early diagnosis of CWP.

(J Occup Health 2014; 56: 301-308)

Key words: Coal workers' pneumoconiosis, Filmscreen radiography, High-resolution computerized tomography, Opacity coalescence, Opacity shape, Profusion of small opacities

Coal workers' pneumoconiosis (CWP), caused by long-term exposure to occupational coal dust, remains one of the most serious occupational diseases in developing countries ${ }^{1)}$. It is also the most serious occupational disease in China. Recent reports indicated that more than 2.65 million workers are exposed to coal mine dust ${ }^{2}$ and about 5,300 to 13,000 incident CWP cases (more than $50 \%$ of all pneumoconiosis) were reported annually from 2007 to 2010 by the Chinese Ministry of Health ${ }^{3)}$. A meta-analysis of published data from 2001 to 2011 found that the pooled prevalence of CWP in China was $6.02 \%$, much higher than that in the UK $(0.8 \%$, during 1998-2000) or USA $(3.2 \% \text { in the } 2000 \mathrm{~s})^{4}$. To detect adverse health effects, Chinese national regulations have mandated periodic medical screening for all workers exposed to hazardous dusts since 1963. These peri- 
odic medical screenings normally include a physical examination and conventional film-screen chest radiography (FSR). Currently, the diagnosis of pneumoconiosis in China is based on radiographic findings on conventional FSR using the Chinese Diagnostic Criteria of Pneumoconiosis ${ }^{5}$. The diagnostic criteria in China and its series of standard radiographic films are largely analogous to the ILO classification system. The agreement regarding detection of the presence of pneumoconiosis between the Chinese and ILO systems was $89.3 \%$ in our previous study ${ }^{6}$. The gold standard diagnostic method for pneumoconiosis including CWP is based on FSR by ILO and many other countries.

The profusion of small opacities and its lung field distribution are the main basis for clinical diagnosis of earlier CWP by FSR. When the disease develops, some small opacities will progress in size or merge together. Therefore, the border of small opacity coalescence is frequently confused in FSR. Blurred small opacities make CWP diagnosis difficult, especially in the early stage. Hence, many workers can not get timely diagnosis. New techniques in detecting nodular pulmonary parenchymal changes are expected.

In recent years, high-resolution computerized tomography (HRCT) has been demonstrated to be more sensitive in detecting nodular pulmonary parenchymal changes and emphysema, and perhaps it could provide more sensitive images for pneumoconiosis diagnosis, as it can reveal fine structural changes in the secondary lobule ${ }^{7-10)}$. HRCT may provide a confident diagnosis without the need for a surgical biopsy ${ }^{11)}$. Furthermore, it can quantify the extent of lung abnormalities and can be used to make up composite indexes that better estimate disease severity and prognosis ${ }^{12,13)}$. It also makes isotropic data acquisition possible, and it allows for multiplane reformation of high-resolution images to visualize lung anatomy and pathology in unprecedented detail. This latter characteristic is thought to be helpful for evaluating pulmonary parenchymal changes of CWP and its complications. The advantages of HRCT in pneumoconiosis diagnosis have garnered more and more attention, and its role in clinical diagnosis of pneumoconiosis is being discussed. Digital direct readout radiography, which has good agreement with conventional FSR, was accepted as diagnostic criteria for pneumoconiosis by the ILO in 2011 14,15). Subsequently, whether HRCT could be used as the diagnostic standard for pneumoconiosis aroused heated debate. More research in this field is needed.

In this study, we performed a comparison between HRCT and FSR images for recognition of the profusion of small opacities in a total of 200 coal mine workers. We also evaluated the possible role of HRCT in CWP diagnosis.

\section{Materials and Methods}

\section{Subjects}

From May 2009 through October 2011, we recruited 97 CWP category 1 dust exposed miners diagnosed by FSR at the hospital of a large coal company. Seventy healthy miners and 37 healthy controls without dust exposure were randomly selected from the same mine. These subjects had similar ages ( \pm 5 years) and durations of employment $( \pm 5$ years $)$ as the case. All subjects were male and without active tuberculosis, acute respiratory infection, asthma and chronic airway diseases, and all of them signed an informed consent form. Each of them underwent FSR and HRCT examinations separately within three months. Also the FSR images from previous examinations (last 3 years) of the same subjects were also collected for evaluation. In this study, we collected 209 FSR images from 204 subjects, of which 6 had more than one image. The study was approved by the institutional review board of the General Hospital of Xishan. Demographic data and characteristics of all subjects, including age, work history including start and stop date of coal dust exposure and cigarette consumption (pack-years) were collected by trained investigators using uniform questionnaires.

The quality of 413 images from FSR and HRCT for the 204 subjects is presented in Table 1. None of the HRCT images were classified as unacceptable. Seven FSR images of 4 subjects including 1 CWP subject were classified as unacceptable, and all of their data were excluded from the subsequent analysis. A total of 200 male subjects was remained.

\section{FSR and HRCT examination}

Radiographic images were taken using a Siemens $800 \mathrm{~mA}$ x-ray machine, with the settings of $125 \mathrm{kV}$ and 2-8 $\mathrm{mAs}$ and an acquisition time of less than $0.1 \mathrm{~s}$. The subjects were placed in the posteroanterior position, and the distance between the focal plane and the film was $180 \mathrm{~cm}$. The thin section $(1.0 \mathrm{~mm}) \mathrm{HRCT}$ scanning was performed with the bone algorithm on three selected sections: upper (aortic arch), middle (carina), and lower thorax (inferior pulmonary vein), respectively, using settings of $120 \mathrm{kV}$ and $140 \mathrm{~mA}$. And HRCT scans were obtained during suspended deep inspiration in the supine position.

\section{FSR evaluation}

FSR is the gold standard for pneumoconiosis diagnosis recommended by the ILO and China. Positive or negative Radiographic diagnoses of CWP were made by a group of three qualified physicians according to the Chinese Diagnostic Criteria of Pneumoconiosis (GBZ70-2009). The diagnostic crite- 
Table 1. Technical quality for images $(n=413)$ from film-screen radiography and high-resolution computerized tomography

\begin{tabular}{lcccccc}
\hline \multirow{2}{*}{ Quality grade } & \multicolumn{2}{c}{ Film-screen radiography } & & \multicolumn{2}{c}{$\begin{array}{c}\text { High-resolution } \\
\text { computerized tomography }\end{array}$} \\
\cline { 2 - 3 } \cline { 5 - 6 } & $\mathrm{n}$ & $\%$ & & $\mathrm{n}$ & $\%$ \\
\hline Good & 145 & 69.4 & & 180 & 88.2 \\
Acceptable, no defects & 40 & & 19.1 & & 16 & 7.8 \\
Acceptable, with some technical defects & 17 & & 8.1 & & 8 & 3.9 \\
Unacceptable & 7 & 3.4 & & 0 & 0 \\
\hline
\end{tabular}

ria and the required series of standard radiographic films are largely analogous to the ILO classification system.

\section{HRCT evaluation}

The evaluation for lung parenchyma profusion category of small opacities, profusion score, shape and coalescence was performed by HRCT after FSR evaluation. Opacity shape in one case was decided by the predominant opacity shape in images and visually classified as rounded or irregular by using the same basic principles as previously defined by the ILO for chest radiographs in the case of occupational fibrotic lung disease ${ }^{16)}$.

Rounded opacities included micronodules and nodules. We defined a micronodule as an opacity with a diameter of less than $7 \mathrm{~mm}$ and a nodule as an opacity with a diameter of 8 to $20 \mathrm{~mm}$, referring to the definitions described by Remy-Jardin and his colleagues $^{17)}$.

To evaluate the severity of pulmonary disease, we used a lung parenchyma profusion category system reported by Bérgin et al. Briefly, the categories were as follows: category $0=$ no definite opacities; category $1=\mathrm{a}$ small number of opacities without disruption of vascular markings; category $2=$ many definite opacities, but with no confluence; category $3=a$ confluence of opacities with disruption of vascular markings; and category $4=a$ confluence of opacities extending over two or more slices that was consistent with progressive massive fibrosis (PMF) on $\mathrm{FSR}^{18)}$. The lungs were divided into six zones (three zones on each side): the upper (above the level of the carina), middle (between the carina and inferior pulmonary veins) and the lower (under the level of the inferior pulmonary veins) areas. All HRCT sections were evaluated according to the above category system, and more than $2 / 3$ involvement of the lung parenchyma in corresponding areas was required for the assigned profusion category. The profusion category for opacities in the six zones of the lung was first recorded separately, and then the highest category in the six zones was used for the subject.

To describe the extent of lung parenchyma opacities, the profusion score was determined by converting the above 0-4 category system to a linear scale of $0-10$, with $0 /-=0,0 / 0=0,0 / 1=1,1 / 0=2,1 / 1=3,1 / 2=4$, $2 / 1=5,2 / 2=6,2 / 3=7,3 / 2=8,3 / 3=9$ and $3 /+=10$. This is similar to the ILO profusion score for chest radiographic evaluation. The profusion scores for each of the six lung zones were calculated first, and then the total profusion score for the six zones was used to represent the pulmonary parenchyma opacity. A set of images from Bérgin for profusion category was used as reference images ${ }^{18)}$.

\section{Images reading protocol}

In the beginning, all FSR and HRCT images were independently and successively assessed by three certified readers for profusion category of small opacities, shape and coalescence in accordance with the relative standards. Two of the three readers were respiratory physicians with national diagnostic qualifications for pneumoconiosis, and the other one was a thoracic radiologist experienced in reading thoracic images. Unless more than one reader had the same interpretation, the images were reviewed by all readers again, and then the final consensus was reached through discussion.

\section{Statistical analysis}

Statistical analysis was performed with the SPSS version 17.0 software (SPSS Inc., Chicago, Il, USA). The sensitivity and false positive rate for HRCT were calculated with the following equations. Diagnosis with FSR was used as the gold standard.

Sensitivity=number positive by the test/number positive by gold standard

False positive rate=number positive by the test/ number negative by gold standard

Kappa statistics were employed to examine the agreement of the results between FSR and HRCT. We categorized kappa values less than 0.2 as poor, 0.2-0.4 as fair, $0.4-0.6$ as moderate, $0.6-0.8$ as moderately 
Table 2. Characteristics of all the subjects

\begin{tabular}{lccccc}
\hline Group & $\begin{array}{c}\text { Number of } \\
\text { workers }\end{array}$ & $\begin{array}{c}\text { Mean age } \\
\text { (years) }\end{array}$ & $\begin{array}{c}\text { Years of } \\
\text { working }\end{array}$ & $\begin{array}{c}\text { Number of } \\
\text { smokers }^{\mathrm{a}}(\%)\end{array}$ & $\begin{array}{c}\text { Smoking amount } \\
(\text { packs } \times \text { years })\end{array}$ \\
\hline Healthy controls & 37 & $46.67 \pm 6.76$ & $25.70 \pm 7.53$ & $26(70.27)$ & $13.89 \pm 13.04$ \\
Healthy coal miners & 67 & $47.12 \pm 7.35$ & $24.49 \pm 7.70$ & $53(79.10)$ & $18.65 \pm 14.78^{*}$ \\
FSR CWP coal miners & 96 & $49.01 \pm 6.16$ & $24.10 \pm 7.63$ & $93^{*, \#(96.88)}$ & $17.13 \pm 14.89^{*}$ \\
\hline
\end{tabular}

FSR, film-screen radiography; CWP, coal workers' pneumoconiosis.

ancluding current and former smokers. ${ }^{*}$ Compared with healthy controls, $p<0.01$. ${ }^{\#}$ Compared with healthy coal miners, $p<0.01$.

Table 3. Profusion categories for all subjects $(n=200)$ by FSR and HRCT*

\begin{tabular}{cccccc}
\hline \multirow{2}{*}{ HRCT } & \multicolumn{5}{c}{ FSR } \\
\cline { 2 - 6 } & $0 / 0$ & $0 / 1$ & $1 / 0,1 / 1,1 / 2$ & $\geq 2 / 1$ & Total \\
\hline $0 / 0$ & 45 & 11 & 0 & 0 & 56 \\
$0 / 1$ & 1 & 2 & 4 & 0 & 7 \\
$1 / 0,1 / 1,1 / 2$ & 5 & 21 & 59 & 4 & 89 \\
$\geq 2 / 1$ & 0 & 3 & 16 & 29 & 48 \\
Total & 51 & 37 & 79 & 33 & 200 \\
\hline
\end{tabular}

FSR, film-screen radiography; HRCT, high-resolution computerized tomography; CI, confidence interval. ${ }^{*} \kappa=0.69$ (95\% CI: $0.63-0.76)$.

high and greater than 0.8 as high. The differences among groups were compared using ANOVA and the Chi-square test, as appropriate. $p<0.05$ was considered statistically significant.

\section{Results}

\section{Study subjects}

Among the 200 subjects, there were 96 CWP category 1 coal miners according to FSR, 67 healthy coal miners without CWP evidence according to FSR and 37 healthy controls. Table 2 shows that there was no statistical difference in mean age among the three groups. The average net years in dust for coal miners with CWP were not different from that for those without CWP. The number of cigarette smokers (including current and former smokers) among the CWP coal miners was significantly higher than in the other two groups $(p<0.01)$.

Comparison of the profusion of small opacities between FSR and HRCT

The agreement with regard to small opacity profusion assessment between FSR and HRCT was good, with a crude agreement rate of $67.5 \%$, a weighted kappa ( $\kappa$ ) of 0.69 and a 95\% CI of 0.63-0.76 (Table 3). Small opacity profusion was similarly distributed between the left and right lungs by zones (Fig. 1). Good agreement was observed between the two meth- ods in the upper and middle zones, and moderate agreement was observed in the lower zones.

\section{Comparison of opacity shape designations between} FSR and HRCT

For coal miners, the majority of opacity shapes were irregular in FSR images of CWP subjects (94/128, $73.44 \%$ ), whereas cases with rounded opacity were predominant in HRCT images $(149 / 158,94.30 \%$ ) (Table 4). The distribution of opacity shapes was statistically significantly different $\left(\chi^{2}=140.82, p<0.01\right)$ between FSR and HRCT.

\section{Lung parenchyma profusion score in FSR and HRCT}

The extent of pulmonary parenchyma opacity was expressed as the mean profusion score (Table 5). The mean profusion score for HRCT $(0.11 \pm 0.46)$ was significantly lower compared with that for FSR $(0.33 \pm 0.76)$ in the healthy controls. However, the mean scores for profusion of small opacities for HRCT were $12.25 \pm 11.52$ in the healthy coal miners and $23.09 \pm 10.05$ in the FSR CWP miners. Both values were statistically significantly higher than those for FSR. The scores for profusion of small opacities for FSR were $2.84 \pm 2.8$ in the healthy coal miners and $18.86 \pm 7.93$ in the FSR CWP miners. The difference in profusion scores between CWP coal miners and healthy controls for HRCT were higher than those for FSR. A statistically significant difference existed between the two methods. Our results indicated that the background profusion scores for HRCT were lower than those for FSR. Coal dust exposure-induced lung parenchyma opacity changes in HRCT were higher than those in FSR.

\section{Evaluation for CWP diagnosis}

For dust-exposed coal workers, the agreement rate for detection of CWP status (positive or negative) was moderately high, with a crude agreement rate of $87.1 \%$ and weighted Kappa value was 0.72 (95\% CI: 0.62-0.83) (Table 6). Furthermore, 93 of the 96 FSR category $1 \mathrm{CWP}$ miners were detected by HRCT. The sensitivity was $96.9 \%$. However, the results of 


\section{Left}
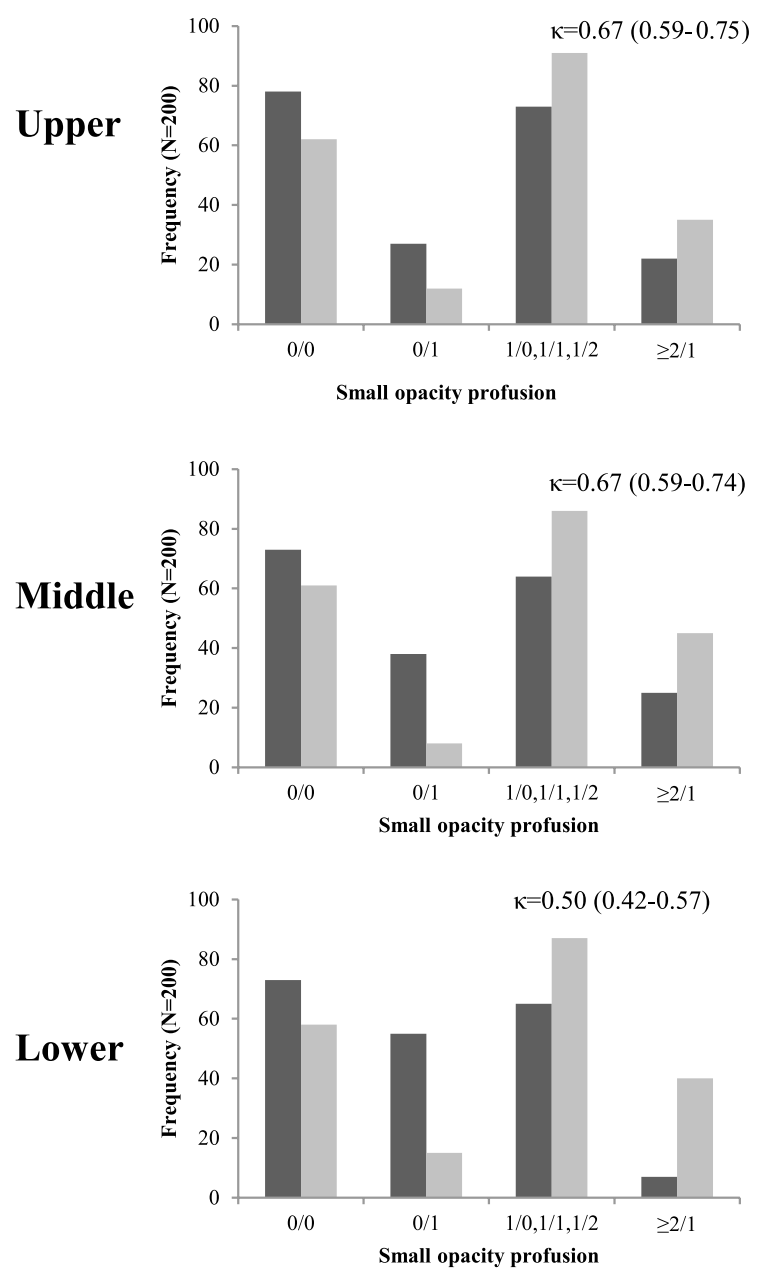

Right
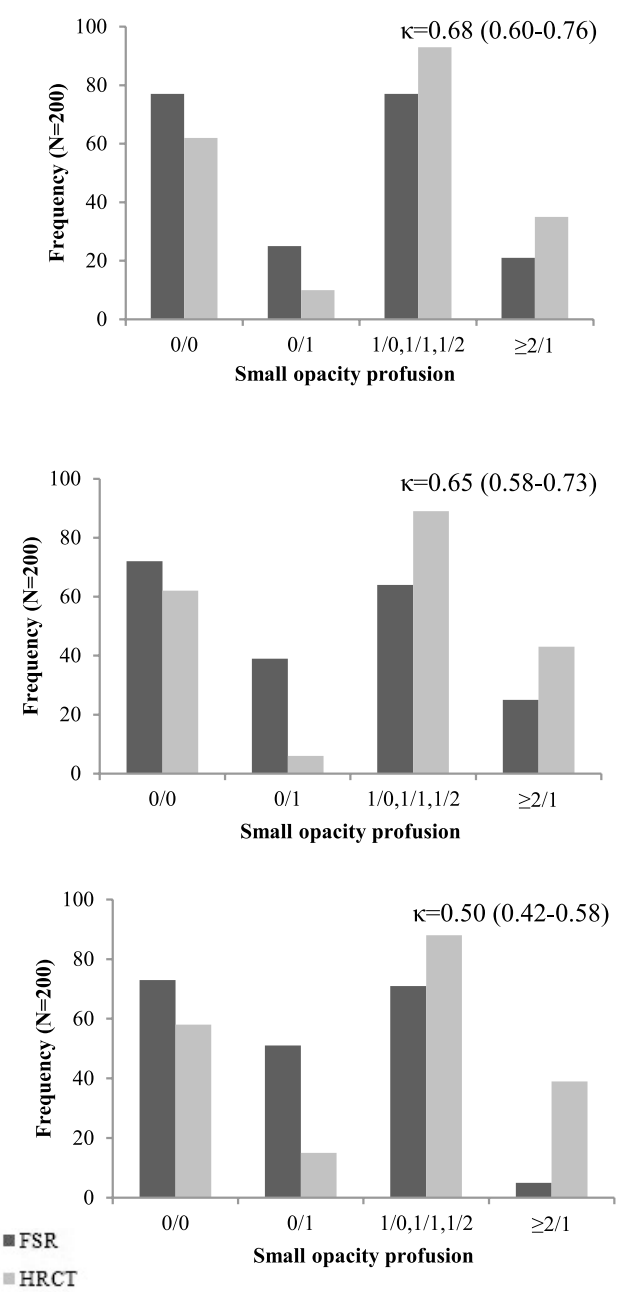

Fig. 1. Profusion of small opacities by lung zone of 200 coal mine workers. FSR, film-screen radiography; HRCT, high-resolution computerized tomography; $\kappa$, weighted kappa value.

Table 4. Comparison of opacity shape distribution between FSR and HRCT

\begin{tabular}{lcccc}
\hline Method & $\begin{array}{c}\text { Number of } \\
\text { coal miners }\end{array}$ & $\begin{array}{c}\text { Opacity } \\
\text { positive cases }\end{array}$ & $\begin{array}{c}\text { Rounded opacity } \\
\text { cases. N }(\%)\end{array}$ & $\begin{array}{c}\text { Irregular opacity } \\
\text { cases. N (\%) }\end{array}$ \\
\hline FSR & 163 & 128 & $34(26.56)$ & $94(73.44)$ \\
HRCT & 163 & 158 & $149(94.30)^{*}$ & $9(5.70)$ \\
\hline
\end{tabular}

FSR, film-screen radiography; HRCT, high-resolution computerized tomography.

*: Compared with FSR, $\mathrm{X}^{2}=140.82, p<0.01$.

Table 5. The profusion scores for small opacities in FSR and HRCT for all the subjects

\begin{tabular}{lcccc}
\hline Group & FSR & HRCT & $t$ & $p$ \\
\hline Healthy controls & $0.33 \pm 0.76$ & $0.11 \pm 0.46$ & -2.09 & 0.043 \\
Healthy coal miners & $2.84 \pm 2.8$ & $12.25 \pm 11.52$ & 8.06 & $<0.0001$ \\
FSR CWP coal miners & $18.86 \pm 7.93$ & $23.09 \pm 10.05$ & 6.87 & $<0.0001$ \\
\hline
\end{tabular}

FSR, film-screen radiography; HRCT, high-resolution computerized tomography. 
Table 6. CWP diagnosis for 163 dust-exposed coal mine workers*

\begin{tabular}{lcc}
\hline \multirow{2}{*}{ HRCT } & \multicolumn{2}{c}{ FSR } \\
\cline { 2 - 3 } & CWP positive & CWP negative \\
\hline CWP positive & 93 & 18 \\
CWP negative & 3 & 49 \\
\hline
\end{tabular}

FSR, film-screen radiography; HRCT, high-resolution computerized tomography; CWP, coal workers' pneumoconiosis; CI, confidence interval. $*_{\kappa}=0.72$ (95\% CI: $0.62-0.83)$.

HRCT indicated that 62 of the $93(64.6 \%)$ cases were category 1 CWP, $30(31.3 \%)$ cases were category 2 CWP and 1 (1\%) cases were category 3 CWP. The other 3 FSR category 1 CWP miners were classified as $0 / 1$ by HRCT.

Among the 67 coal miners negative by FSR, 49 miners were classified as $0 / 0$ or $0 / 1$, and 18 miners were classified as category 1 CWP by HRCT. The false positive rate was $26.9 \%$.

\section{Discussion}

In present study, we found that agreement regarding detection of the presence of pneumoconiosis between FSR and HRCT was $87.1 \%$. HRCT could detect 96.9\% pneumoconioses diagnosed by FSR, and HRCT was more sensitive than FSR in recognition of the profusion of small opacities in coal miners. These results suggested that HRCT had clinical diagnosis value. At the same time, we noted that 18 of the $67(26.9 \%)$ miners who were CWP negative by FSR were classified as category 1 by HRCT. This result indicates that the role of HRCT in pneumoconiosis diagnosis in the early stage needs further evaluation.

Although the current diagnostic criteria for CWP are based on FSR, which is recommended as the gold standard by the ILO and many countries, it is well known that FSR has several limitations for pneumoconiosis diagnosis. For example, some opacities will appear as superimposed structures, and the technical quality of FSR images is sometimes not very good. In particular, when there are low profusion scores in FSR, it is difficult to distinguish $1 / 0$ from $0 / 1$, and the interpretation would be rather subjective. Welch et al. reported that the interobserver variability for the detection of radiological signs of early asbestosis and pleural fibrosis was lower than expected ${ }^{19}$. When FSR was ambiguous, HRCT was reported to be more sensitive than FSR in the detection of diffuse lung parenchyma diseases ${ }^{10,20-22)}$. Sun et al. observed that 8 subjects were diagnosed as having early silicosis based on HRCT of 30 subjects whose diagnosis were non-silicosis on FSR according to the same criteria ${ }^{23}$.
Their results were similar to ours. The study of Remy-Jardin also reported that 11 of 48 coal miners without CWP according to FSR (ILO profusion score $<1 / 0$ ) had CT features of CWP (CT profusion score $>1 / 0)^{17)}$. The false positive rate $(22.9 \%)$ in that study was a little lower than ours $(26.9 \%)$.

There are two reasons why more pneumoconiosis cases were diagnosed by HRCT scans. On the one hand, with its high resolution, HRCT is superior to FSR for the detection of small opacities, interstitial fibrosis and emphysema, especially in low-grade pneumoconiosis ${ }^{24)}$. Even though there are no pathognomonic radiographic signs of dust exposure, specific HRCT signs can be recognized like a ground-glass appearance, bullae-bleb and emphysema ${ }^{25}$. On the other hand, HRCT can scan all the lung tissue in a single breath hold. During this short time, the variability in the degree of inspiration can be greatly minimized. As a result, CWP-associated nodules appear with sharper borders, and they are easier to recognize by HRCT than by FSR. This indicated that HRCT can reveal pneumoconiosis cases earlier than FSR. Several studies have found that coal miners negative by FSR but positive by HRCT had a recognized histological abnormality in their lungs ${ }^{24-26}$. The role of HRCT in pneumoconiosis diagnosis in the early stage and whether HRCT can be used as diagnostic criteria for pneumoconiosis need further evaluation.

The results of this study provided evidence for the advantages of HRCT scans in pneumoconiosis diagnoses. Firstly, the agreement regarding pneumoconiosis status (positive or negative) between the two methods was high $(87.1 \%)$. Ninety-three of the 96 miners CWP positive by FSR were evaluated as positive by HRCT; the sensitivity was very high. Only 3 FSR pneumoconiosis cases were missed. We noticed that ground-glass attenuation and mixed emphysema, which might have obscured nodules in images, were found in all 3 cases when all their scans were read together. This made it difficult to distinguish CWP from nonoccupational fibrotic lung diseases such as IPF (idiopathic pulmonary fibrosis). This phenomenon was reported in a previous study ${ }^{27}$. Secondly, HRCT appeared better for recognition nodule coalescence. Thirdly, coal dust exposure induced a large difference or change in lung parenchyma opacity profusion scores in HRCT compared with those in FSR. Profusion scores represented the extent of pulmonary parenchyma opacities, which was the main pathological change of CWP. Therefore, HRCT is more sensitive for identification of the profusion of small opacities which could not be distinguished by FSR.

It was interesting that the majority of CWP-associated opacities manifested as rounded 
nodules in HRCT $(149 / 158,94.30 \%)$ scans, whereas the majority of opacities manifested as irregular opacities in FSR, and only 34 (26.56\%) manifested as rounded nodules. The difference between the two methods was statistically significant $\left(\chi^{2}=140.82\right.$, $p<0.01)$. This phenomenon was mainly considered to be due to reduced superimposition and increased isolation achieved by HRCT. In this situation, readers could identify CWP-associated opacities with more confidence and less hesitation.

One potential limitation of this study is that we did not include category 2 pneumoconiosis or higher in FSR. The reason for this is that we hoped to compare earlier changes in pneumoconiosis between FSR and HRCT. Also most of patients with progressed CWP would not accept more radiographic examinations.

The results of this study indicated that the agreement regarding detection of the presence of pneumoconiosis between FSR and HRCT was $87.1 \%$. The HRCT scans were more sensitive than FSR in recognition of the profusion of small opacities among coal miners. About $26.9 \%$ miners negative for CWP by FSR were classified as positive for CWP by HRCT, suggesting that more research is needed to evaluate the role of HRCT in early diagnosis of CWP.

Acknowledgments: This work was financially supported by grants from the National Basic Research Program of China (2011CB503804, 2011CB512102). We are indebted to Dr. Meilin Wang at the US National Institution for Occupational Safety and Health for providing professional advice and valuable comments.

\section{References}

1) Chen W, Liu Y, Wang H, et al. Long-term exposure to silica dust and risk of total and cause-specific mortality in chinese workers: a cohort study. PLoS Med 2012; 4: e1001206.

2) The 57,000 Chinese coal miners suffer from lung disease annually. [Online]. 2010 [cited 2010 Nov 11]; Available from : URL: http://english.peopledaily.com.cn/90001/90782/7196279.html

3) Ministry of Health of the People's Republic of China. Chinese Annual Health Statistical Report in 2010. Beijing.

4) Mo J, Wang L, Au W, Su M. Prevalence of coal workers' pneumoconiosis in China: a systematic analysis of 2001-2011 studies. Int J Hyg Environ Health 2013.

5) Ministry of Health of PR China. Diagnostic criteria of pneumoconiosis (GBZ 70-2009). 2009.

6) Hodous TK, Chen RA, Kinsley KB, et al. A comparison of pneumoconiosis interpretation between Chinese and American readers and classifications. J Tongji Med Univ 1991; 4: 225-9.

7) Arakawa H, Fujimoto K, Honma K, et al.
Progression from near-normal to end-stage lungs in chronic interstitial pneumonia related to silica exposure: long-term CT observations. AJR Am J Roentgenol 2008; 4: 1040-5.

8) Arakawa H, Johkoh $\mathrm{T}$, Honma $\mathrm{K}$, et al. Chronic interstitial pneumonia in silicosis and mix-dust pneumoconiosis: its prevalence and comparison of CT findings with idiopathic pulmonary fibrosis. Chest 2007; 6: 1870-6.

9) Mazziotti S, Gaeta M, Costa C, et al. Computed tomography features of liparitosis: a pneumoconiosis due to amorphous silica. Eur Respir J 2004; 2: 208-13.

10) Mosiewicz J, Myslinski W, Zlomaniec G, CzabakGarbacz R, Krupski W, Dzida G. Diagnostic value of high resolution computed tomography in the assessment of nodular changes in pneumoconiosis in foundry workers in Lublin. Ann Agric Environ Med 2004; 2: 279-84.

11) Sverzellati N. Highlights of HRCT imaging in IPF. Respir Res 2013: S3.

12) Sumikawa H, Johkoh T, Colby $T$ V, et al. Computed tomography findings in pathological usual interstitial pneumonia: relationship to survival. Am J Respir Crit Care Med 2008; 4: 433-9.

13) Best AC, Meng J, Lynch AM, et al. Idiopathic pulmonary fibrosis: physiologic tests, quantitative CT indexes, and CT visual scores as predictors of mortality. Radiology 2008; 3: 935-40.

14) International $\mathrm{L} O$, Guidelines for the use of the ILO International Classification of Radiographs of Pneumoconioses (Revised edition 2011). 2011.

15) Mao L, Laney AS, Wang ML, et al. Comparison of digital direct readout radiography with conventional film-screen radiography for the recognition of pneumoconiosis in dust-exposed Chinese workers. J Occup Health 2011; 5: 320-6.

16) Begin R, Ostiguy G, Filion R, Colman N, Bertrand P. Computed tomography in the early detection of asbestosis. Br J Ind Med 1993; 8: 689-98.

17) Remy-Jardin M, Degreef J M, Beuscart R, Voisin C, Remy J. Coal worker's pneumoconiosis: CT assessment in exposed workers and correlation with radiographic findings. Radiology 1990; 2: 363-71.

18) Bergin CJ, Muller NL, Vedal S, Chan-Yeung M. CT in silicosis: correlation with plain films and pulmonary function tests. AJR Am J Roentgenol 1986; 3: 477-83.

19) Welch LS, Hunting KL, Balmes J, et al. Variability in the classification of radiographs using the 1980 international labor organization classification for pneumoconioses. Chest 1998; 6: 1740-8.

20) Lopes AJ, Mogami R, Capone D, Tessarollo B, de Melo PL, Jansen JM. High-resolution computed tomography in silicosis: correlation with chest radiography and pulmonary function tests. J Bras Pneumol 2008; 5: 264-72.

21) Levin SM, Kann PE, Lax MB. Medical examination for asbestos-related disease. Am J Ind Med 2000; 1 : 6-22. 
22) Altin R, Savranlar A, Kart L, et al. Presence and HRCT quantification of bronchiectasis in coal workers. Eur J Radiol 2004; 2: 157-63.

23) Sun J, Weng D, Jin C, et al. The value of high resolution computed tomography in the diagnostics of small opacities and complications of silicosis in mine machinery manufacturing workers, compared to radiography. J Occup Health 2008; 5: 400-5.

24) Meijer E, Tjoe Nij E, Kraus T, et al. Pneumoconiosis and emphysema in construction workers: results of HRCT and lung function findings. Occup Environ Med 2011; 7: 542-6.

25) Savranlar A, Altin R, Mahmutyazicioglu K, et al. Comparison of chest radiography and highresolution computed tomography findings in early and low-grade coal worker's pneumoconiosis. Eur J Radiol 2004; 2: 175-80.

26) Remy-Jardin M, Remy J, Artaud D, Deschildre F, Duhamel A. Diffuse infiltrative lung disease: clinical value of sliding-thin-slab maximum intensity projection CT scans in the detection of mild micronodular patterns. Radiology 1996; 2: 333-9.

27) Shida H, Chiyotani K, Honma K, et al. Radiologic and pathologic characteristics of mixed dust pneumoconiosis. Radiographics 1996; 3: 483-98. 